\title{
A changing journal in a changing health care
}

\author{
Mario Eandi ${ }^{1}$ \\ 1 Department of Pharmacology, University of Torino, Italy
}

Almost 11 years ago, Farmeconomia e percorsi terapeutici was launched as the first Italian journal about pharmacoeconomics. When it was launched, the journal aimed at being a reference in this field in the Italian setting where concepts and terminology of economic evaluations were quite new.

Over the past decade, many things have changed.

The health care systems, created during a period of relative prosperity in the developed world, are now facing the effects of the global financial slowdown. Economic aspects are becoming even more important. Across Europe, public expenditure is always increasing, and new methods to balance budgets and restrain spending are needed. Reflections on ways to generate new resources, inspired by equity and efficiency principles, are essential for all the stakeholders.

The business climate for pharmaceutical companies has consistently changed as well, both in structure and strategies. We assisted on the one hand to the merging of many companies in few Big Pharma, and on the other hand to the launch of highly-innovative start-ups.

The companies are facing the breaking out of the blockbuster mentality and the challenges related to the generication of their blockbuster products. Nonetheless, developing new medicines is more important than ever before, and investments in new technologies offer new ways to improve the medicines development process and foster innovation. R\&D strategies of new drugs are increasingly oriented towards individualized therapies, use of biomarkers, niche indications and orphan diseases. Finally, the rising cost of new drugs represents a threat to the financial sustainability of the welfare and health care system even of the richest countries.

Also pharmacoeconomics has grown and changed rapidly in recent years, with a wider use of analyses based on controlled clinical trials and observational studies, the use of advanced techniques of modelling and simulation (DES-Discrete Event Simulation, MABS-Multi Agent Based Simulation), and the integration of CEA, CUA and BIM techniques with HTA processes.
We still believe that economic aspects are essential to build a well-functioning health care system, in order to develop governance policies that ensure equitable access to essential medical products, vaccines and technologies, and people's access to needed services.

Nonetheless, we perceive the inherent limitations of the predominantly utilitarian approach and the necessity to overcome the limitations of the standard CEA and CUA analyses. Prioritizing the appropriateness analysis for each patient is the final goal to optimize not only health care outcomes, but also the economic balance of health care systems.

In this new scenario, we felt the need to rethink our journal. Starting from this issue of Farmeconomia - Health economics and therapeutic pathways, our intention will be to wide the journal contents, publishing articles that reflect current discussions and priorities in health economics. To this purpose, we enlarged the scientific board, involving many experts among different countries across the world. The scientific board reflects the idea of authors and readers we'd like to reach: clinicians and researchers in various countries who wish to exchange national and international experiences and research results about the economic factors that affect the evolution of their health care systems. The final aim of our journal will be to provide an overview of the Italian health care system to non-Italian readers, while spreading in Italy the experiences of other countries and settings.

This new approach to re-defining the Farmeconomia mission has allowed us to capitalize on one of our key underlying values: open access. By access to its articles by all readers, the journal aims at spreading the knowledge about economics topics, incentivising the debate among readers.

No doubt it is a big challenge. However, it is certainly worthy to compare different situations, increasing the dialogue in academic and scientific community.

We hope such changes and innovations will stimulate renewed interest to a wider readership of researchers, stakeholders and decision makers.

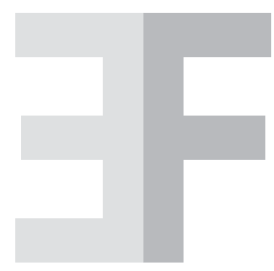

EDITORIAL

( 